\title{
Radiation Dose Reduction by Indication-Directed Focused z-Direction Coverage for Neck CT
}

\author{
(1)A.K. Parikh and ${ }^{\circledR}$ C.C. Shah
}

\begin{abstract}
BACKGROUND AND PURPOSE: The American College of Radiology-American Society of Neuroradiology-Society for Pediatric Radiology Practice Parameter for a neck CT suggests that coverage should be from the sella to the aortic arch. It also recommends using CT scans judiciously to achieve the clinical objective. Our purpose was to analyze the potential dose reduction by decreasing the scan length of a neck CT and to assess for any clinically relevant information that might be missed from this modified approach.
\end{abstract}

MATERIALS AND METHODS: This retrospective study included 126 children who underwent a neck CT between August 1, 2013, and September 30, 2014. Alteration of the scan length for the modified CT was suggested on the topographic image on the basis of the indication of the study, with the reader blinded to the images and the report. The CT dose index volume of the original scan was multiplied by the new scan length to calculate the dose-length product of the modified study. The effective dose was calculated for the original and modified studies by using age-based conversion factors from the American Association of Physicists in Medicine Report No. 96.

RESULTS: Decreasing the scan length resulted in an average estimated dose reduction of $47 \%$. The average reduction in scan length was $10.4 \mathrm{~cm}$, decreasing the overall coverage by $48 \%$. The change in scan length did not result in any missed findings that altered management. Of the 27 abscesses in this study, none extended to the mediastinum. All of the lesions in question were completely covered.

CONCLUSIONS: Decreasing the scan length of a neck CT according to the indication provides a significant savings in radiation dose, while not altering diagnostic ability or management.

ABBREVIATIONS: AAPM = American Association of Physicists in Medicine; ACR-ASNR-SPR = American College of Radiology-American Society of NeuroradiologySociety for Pediatric Radiology; CTDI $_{\text {vol }}=$ CT dose index volume; DLP = dose-length product

$\mathbf{N}$ umerous conditions can occur within the pediatric neck prompting an imaging request, commonly a CT scan. These include, but are not limited to, tonsillar disease, odontogenic infection, congenital anomalies, and neoplastic conditions. The history and physical examination in the symptomatic child can be difficult and limited, rendering the use of imaging pivotal. ${ }^{1} \mathrm{CT}$ is vital in teasing out the many varying conditions of the pediatric extracranial head and neck that will often present in an acute care setting. ${ }^{1}$ The American College of Radiology-American Society of

Received August 24, 2015; accepted after revision November 24.

From the Department of Radiology (A.K.P.), Mayo Clinic, Jacksonville, Florida; and Department of Radiology (C.C.S.), Nemours Children's Specialty Care, Wolfson's Children's Hospital, Jacksonville, Florida.

Paper previously presented at: Society for Pediatric Radiology Annual Meeting, April 27 to May 1, 2015; Bellevue, Washington. The first and corresponding author was given the John Kirkpatrick Young Investigator Award for research in the above project.

Please address correspondence to Ashishkumar K. Parikh, MD, 807 Children's Way, Jacksonville, FL 32207; e-mail: ashishckt@gmail.com; @ashishckt

三 Indicates article with supplemental on-line table.

http://dx.doi.org/10.3174/ajnr.A4672
Neuroradiology-Society for Pediatric Radiology (ACR-ASNRSPR) practice parameter for the performance of CT of the extracranial head and neck suggests that coverage should be through the area of interest, specifically from the skull base (sella floor) to the top of the aortic arch. ${ }^{2}$

While providing simplicity, a neck CT with "blanket" coverage irrespective of indication is contrary to the principles of as low as reasonably achievable in reducing or optimizing the radiation dose. ${ }^{3}$ The principle of as low as reasonably achievable is paramount because recent studies have demonstrated a small but significant increase in cancer incidence among children exposed to ionizing radiation. ${ }^{4}$ Furthermore, it is well-documented that infants and children are much more vulnerable to the effects of radiation exposure, given their longer life span and increased sensitivity to radiation-induced cancers than their adult counterparts. ${ }^{5}$ Given that approximately 7 million CT scans are performed annually in children in the United States, any attempts at dose reduction can have a positive impact. ${ }^{6}$

By decreasing the scan length (z-axis) to the clinical indication, unnecessary radiation exposure can be eliminated. Our purpose was 
Table 1: Demographics

\begin{tabular}{lrccr}
\hline & Mean & Median & Range & \multicolumn{1}{c}{ SD } \\
\hline Age (mo) & 99.4 & 85.0 & $2-216$ & 66.6 \\
Age (yr) & 8.3 & 7.1 & $0-18$ & 5.5 \\
Height $(\mathrm{cm})$ & 125.7 & 125.0 & $55-190$ & 35.6 \\
Weight $(\mathrm{kg})$ & 36.7 & 25.5 & $5-128$ & 27.9 \\
\hline
\end{tabular}

Table 2: Technique

\begin{tabular}{lc}
\hline \multicolumn{1}{c}{ Parameters } & Setting \\
\hline Kilovolt & $100-140$ \\
Milliampere (min) & 79 \\
Milliampere (max) & 300 \\
Detector & $0.625 \mathrm{~mm}$ \\
CT scanner & 64 section \\
Section thickness & $2.5 \mathrm{~mm}$ \\
Gantry rotation time & 0.5 seconds \\
Pitch & 0.96875 \\
\hline
\end{tabular}

Note:-Min indicates minimum; Max, maximum.

to analyze the potential dose reduction by decreasing the $z$-axis length of neck CT scans and to assess for any clinically relevant information that might be missed from this modified approach.

\section{MATERIALS AND METHODS}

This retrospective study included 126 children 18 years of age or younger who underwent a neck CT on a 64-section CT scanner (LightSpeed VCT; GE Healthcare, Milwaukee, Wisconsin) at a tertiary care children's hospital between August 1, 2013, and September 30, 2014. Wolfson's Children's Hospital/Nemours Children's Speciality Care institutional review board approval was obtained, and informed consent was waived for this Health Insurance Portability and Accountability Act-compliant retrospective study. The cohort studied comprised 60 girls and 66 boys, with age ranges from 2 months to 18 years, with a mean age of 8.3 years. The average weight and height within the cohort analyzed were $37 \mathrm{~kg}$ (range, 5-128 kg) and $126 \mathrm{~cm}$ (range, 55-190 cm), respectively (Table 1 ). No children were included who had a combined neck, chest, abdomen, and pelvis CT for an oncologic diagnosis or surveillance. In the oncologic setting, altering the length in the craniocaudad dimension would not be indicated, given the need for complete coverage to assess sites of disease spread. Two children for whom the dose information was not available were excluded.

Helical acquisitions were obtained. Imaging parameters were as follows: 100-140 kV; Automatic Exposure Control; $64 \times 0.625$ $\mathrm{mm}$ detector; gantry rotation time, 0.5 seconds; pitch, 0.96875 ; and a soft-tissue reconstruction algorithm (Table 2). Iterative reconstruction was available on our CT scanner. The range in kilovolts was due to variations in weight, with heavier patients scanned with $140 \mathrm{kV}$ and lighter patients scanned with $100 \mathrm{kV}$. For instance, 6 patients were on the lighter side, weighing $<22.5$ $\mathrm{kg}$ with a set kilovolt of 100 . Two patients were scanned with a kilovolt of 140 because they weighed $89 \mathrm{~kg}$ and $128 \mathrm{~kg}$, respectively. The remaining 118 patients were scanned with the standard set kilovolt of 120 (Table 3). With Automatic Exposure Control, the maximum milliampere was 350 and varied with patient weight (Table 3). The minimum milliampere was 79 for all the patients. In children weighing $<22.5 \mathrm{~kg}$, the maximum milliampere was 200 . From 22.5 to $31.5 \mathrm{~kg}$, the maximum milliampere was 250 . The maximum milliampere was 300 for children weigh-
Table 3: Weight-adjusted tube current and kilovolt

\begin{tabular}{cccc}
\hline Weight $(\mathbf{k g})$ & $\begin{array}{c}\text { Min } \\
\text { Milliampere }\end{array}$ & $\begin{array}{c}\text { Max } \\
\text { Milliampere }\end{array}$ & Kilovolt \\
\hline$<22.5$ & 79 & 200 & 100 \\
$22.5-31.5$ & 79 & 250 & 120 \\
$31.5-40.5$ & 79 & 300 & 120 \\
$\geq 40.5$ & 79 & 350 & 140 \\
\hline
\end{tabular}

Note:-Min indicates minimum; Max, maximum.

ing between 31.5 and $40.5 \mathrm{~kg}$. In children weighing greater than $40.5 \mathrm{~kg}$, the maximum milliampere was 350 .

Among all the included patients, we analyzed the following data points: indication, z-start of the scan (beginning or cranial extent), z-end of the scan (terminal or caudal extent), CT dose index volume $\left(\mathrm{CTDI}_{\mathrm{vol}}\right)$ (milligray), dose-length product (DLP) (milligray $X$ centimeter, based on a $16-\mathrm{cm}$ phantom), modified $\mathrm{z}$-start of the scan, modified z-end of the scan, kilovolt, minimum milliampere, maximum milliampere, milliseconds, patient weight (kilograms), patient height (centimeters), amount of iodinated contrast (milliliter), sex, finding/diagnosis, any findings that would have been missed due to modification of the z-axis, clinical relevance of the missed finding, follow-up method of the main/diagnostic finding, follow-up imaging/pathology of the main finding (if any), age in years and months, total scan length (centimeter), modified scan length (centimeter), modified DLP (milligray $\times$ centimeter), weighting factor, effective dose (millisievert), modified effective dose (millisievert), percentage reduction in craniocaudad length (centimeter) for the modified scans, and percentage reduction in dose from the z-axis modification. Please refer to the Appendix for an explanation of the terminology used in this article.

The investigator (A.K.P., 3 years' experience) was initially presented with the indication of the study and the topographic (scout) image while blinded to the cross-sectional and reformatted CT images and the CT report. On the basis of this information, the investigator suggested an alteration of the z-axis for the modified CT scan (Figure). The z-start/end level and modified z-start/ end level were based on the topographic image (eg, beginning at C4 instead of the sella). Each modification of the z-axis length was individualized to the examination indication in its cranial and caudal extent. However, in cases of a suspected abscess, the z-axis length was modified to begin at the temporomandibular joint and end at the sternoclavicular junction. In cases of "lumps and bumps," the scan length covered several centimeters above and below the lesion in question. For instance, for a palpable lesion of $<2 \mathrm{~cm}$, the marker would be placed at the epicenter of the abnormality and the scan would be performed $3 \mathrm{~cm}$ above and below the marker. For lesions of $>2 \mathrm{~cm}$, the marker would be placed at the cranial and caudal margins of the lesions and the scan would be performed $3 \mathrm{~cm}$ above and below the marker.

"Missed" findings were abnormalities noted on the original CT scan that would no longer be seen due to the reduced $\mathrm{z}$-axis coverage (ie, if the $\mathrm{z}$-axis were modified according to the indication). The clinical relevance of this missed finding was determined by examining the patient's chart to determine whether the initial management would have been altered on the basis of the missed finding. The follow-up method of the diagnostic finding was either clinical or surgical. If the follow-up method was surgical treatment of the offending lesion, the pathology report was reviewed. 


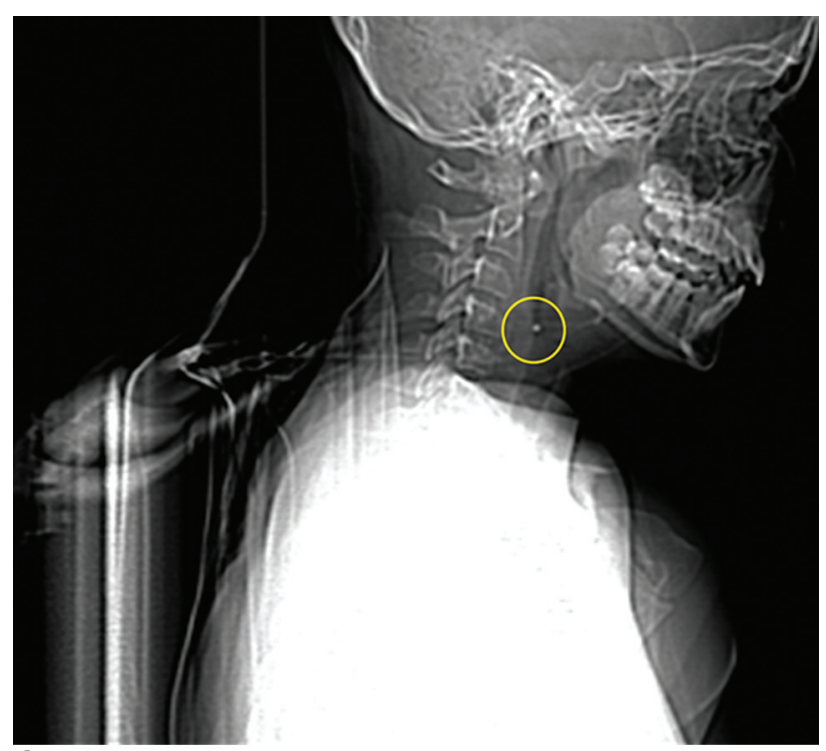

A

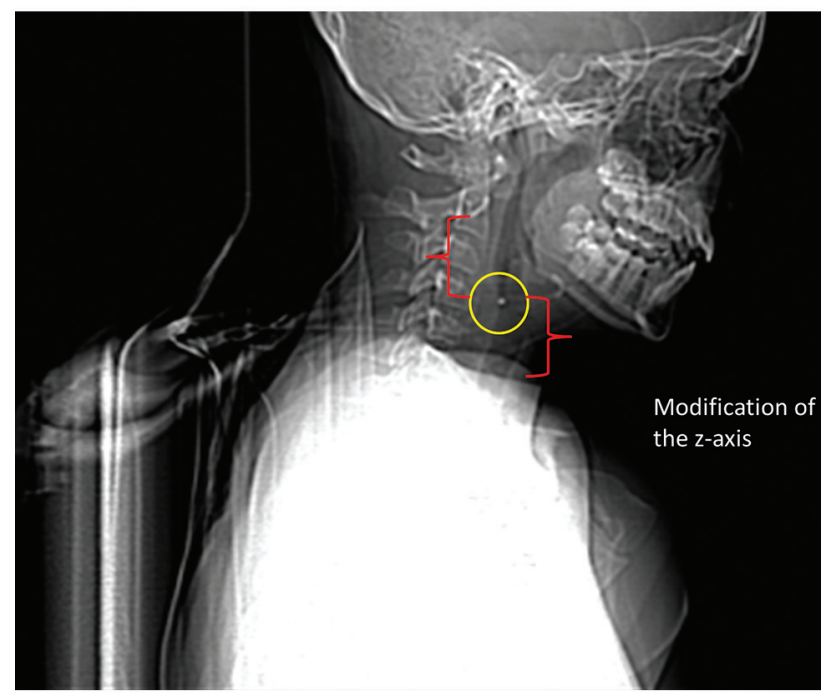

B

FIGURE. Two topographic images of the same patient demonstrate a radiopaque marker at the epicenter of a lesion (yellow circle). The modification of the z-axis is then performed on the basis of the initial clinical indication of a palpable abnormality in the second image (red brackets).

Dose-length product for the modified study was extrapolated from the DLP of the original examination by factoring for the scan length. The modified DLP was calculated by multiplying the modified scan length (centimeters) by the $\mathrm{CTDI}_{\mathrm{vol}}$.

Modified DLP $(\mathrm{mGy})=\mathrm{CTDI}_{\mathrm{vol}}\left(\mathrm{mGy}^{*} \mathrm{~cm}\right) \times$

modified $\mathrm{z}$-axis length $(\mathrm{cm})$.

The effective dose was calculated from the DLP for the original examination and for the modified study by using the conversion factors (Table 4) for various age groups provided in the American Association of Physicists in Medicine (AAPM) Report No. 96 (2008). ${ }^{7}$ These conversion factors were multiplied by the DLP to get the effective dose for the original and modified DLP. The MannWhitney $U$ test was used to determine statistically significant differences between the 2 groups (modified and original scans).
Table 4: Age-specific conversion factors $\left[\kappa\left(\mathrm{mSv} \times \mathrm{mGy}^{-1} \times\right.\right.$ $\left.\left.\mathrm{cm}^{-1}\right)\right]^{\mathrm{a}}$

\begin{tabular}{lccccc}
\hline & 0 Years & 1 Year & 5 Years & 10 Years & Adult \\
\hline Neck & 0.017 & 0.012 & 0.011 & 0.0079 & 0.0059 \\
\hline${ }^{a}$ AAPM No. $96(2008)$. & & & &
\end{tabular}

Table 5: Radiation dose

\begin{tabular}{llllc}
\hline & Mean & Median & Range & SD \\
\hline Z-length $(\mathrm{cm})$ & 20.18 & 20.13 & $5.2-34.0$ & 52.2 \\
DLP $(\mathrm{mGy} \times \mathrm{cm})$ & 111.5 & 69.2 & $20-656$ & 97.6 \\
Modified z-length $(\mathrm{cm})$ & 10.43 & 10.00 & $1.8-20.8$ & 5.16 \\
Modified DLP $(\mathrm{mGy} \times \mathrm{cm})$ & 59.2 & 35.1 & $5-363$ & 60.0 \\
Effective dose $(\mathrm{mSv})$ & 1.02 & 0.78 & $0.25-5.18$ & 0.71 \\
Modified effective dose $(\mathrm{mSv})$ & 0.54 & 0.42 & $0.05-2.86$ & 0.45 \\
Dose reduction $(\%)$ & 47 & 43 & $15-93$ & 20 \\
\hline
\end{tabular}

\section{RESULTS}

Data comparing the DLP and the effective dose for the original and modified examinations are described in Table 5. Briefly, the mean DLP for the original examinations was $111.5 \pm 97.6 \mathrm{mGy} \times$ $\mathrm{cm}$ (range, 20-656 $\mathrm{mGy} \times \mathrm{cm}$; median, $69.2 \mathrm{mGy} \times \mathrm{cm}$ ). The mean DLP for the modified examinations was $59.2 \pm 60.0 \mathrm{mGy} \times$ $\mathrm{cm}$ (range, 5.0-362.5 $\mathrm{mGy} \times \mathrm{cm}$; median, $35.1 \mathrm{mGy} \times \mathrm{cm}$ ). The mean effective dose for the original examinations was $1.02 \pm 0.71$ $\mathrm{mSv}$ (range, $0.25-5.18 \mathrm{mSv}$; median, $0.78 \mathrm{mSv}$ ). In contrast, the mean effective dose for modified examinations was $0.54 \pm 0.45$ $\mathrm{mSv}$ (range, 0.05-2.86 mSv; median, $0.42 \mathrm{mSv}$ ). Decreasing the longitudinal scan length along the $\mathrm{z}$-direction resulted in an average estimated dose reduction of $47 \%$ (range, $1.02-0.54 \mathrm{mSv}$ ) and a median dose reduction of $43 \%$ (range, $0.78-0.42 \mathrm{mSv}$ ). The Mann-Whitney $U$ test demonstrated the differences between the original and modified examinations to be statistically significant with a $P$ value $<.0001$.

The estimated mean effective dose reduction was $53 \%$ in children younger than 1 year of age and $46 \%$ for children between 10 and 18 years of age. Furthermore, there was an average reduction in scan length from the original to the modified examinations of approximately $10.4 \mathrm{~cm}$, which decreased the overall coverage by an average of $48 \%$ in the modified scans. Additional data regarding dose reduction among different age groups in our study are listed in detail in Table 6.

There were no clinically relevant "missed" findings by using the modified CT approach (On-line Table). Most of the abnormalities that would not have been identified with the modified z-length were various congenital anomalies (often vascular) that were not relevant for the clinical indication. One patient had multiple abnormalities involving the head and neck, but none were new findings to the clinicians, confirmed with a search of the patient's electronic medical records. None of the 27 abscesses encountered extended to or involved the mediastinum.

\section{DISCUSSION}

As mentioned previously, the current guidelines adopted by the ACR-ASNR-SPR for the performance of CT of the extracranial head and neck suggest that coverage should be through the area of interest, specifically from the floor of the sella to the top of the aortic arch. This coverage can extend to involve the aortopulmonary window if left recurrent laryngeal nerve pathology is sus- 


\begin{tabular}{|c|c|c|c|c|c|}
\hline & $\begin{array}{l}\text { Younger Than } \\
1 \text { Year }\end{array}$ & $\begin{array}{c}1 \text { Year to Younger } \\
\text { Than } 5 \text { Years }\end{array}$ & $\begin{array}{l}5 \text { Years to Younger } \\
\text { Than } 10 \text { Years }\end{array}$ & $\begin{array}{c}10 \text { Years to Younger } \\
\text { Than } 18 \text { Years }\end{array}$ & $\begin{array}{c}\text { All } \\
\text { Children }\end{array}$ \\
\hline No. of children & 7 & 42 & 27 & 50 & 126 \\
\hline Mean original z-length (cm) & 13.91 & 15.93 & 19.94 & 24.77 & 20.18 \\
\hline Mean modified z-length (cm) & 6.63 & 8.04 & 10.32 & 13.04 & 10.43 \\
\hline \% Decrease in mean z-length for modified CT & 52 & 50 & 48 & 47 & 48 \\
\hline Mean effective dose for original CT (mSv) & 0.86 & 0.63 & 0.73 & 1.53 & 1.02 \\
\hline Mean effective dose for modified CT (mSv) & 0.41 & 0.32 & 0.38 & 0.83 & 0.54 \\
\hline \% Decrease in mean effective dose for modified CT & 53 & 49 & 48 & 46 & 47 \\
\hline
\end{tabular}

pected. ${ }^{2}$ However, the ACR-ASNR-SPR guidelines also recommend using CT scans judiciously to achieve the clinical objective.

Standard neck CT protocols in adults and children often extend to the aortic arch, imaging the superior mediastinum. Although not specifically mentioned with the ACR-ASNR-SPR practice guidelines, the mediastinal coverage is presumed to evaluate mediastinal pathology, often to determine the mediastinal extent of retropharyngeal abscesses. In children, mediastinal abscesses are very rare, especially with the advent of widespread antibiotic treatment. ${ }^{8}$ When mediastinal abscesses do occur, they are most often following iatrogenic or traumatic causes (ie, following thoracic surgery or esophageal perforation). ${ }^{8}$ According to a publication by Tercier et al, ${ }^{8}$ there have been only 8 reported cases in the literature of nontraumatic mediastinal abscesses in children within the 15 years preceding the publication of the article (1989-2004). The rarity of nontraumatic mediastinal abscesses is reinforced by Kumar et $\mathrm{al}^{9}$ as being "extremely uncommon in childhood, especially since the advent of widespread antibiotic treatment." Kumar et al reported approximately 13 cases in the past 2 decades.

This study has demonstrated that the change in scan length would not have resulted in missed findings that could alter patient management. This is important, particularly in situations in which a neck CT is performed for the evaluation of a suspected abscess, a common scenario. With the modified approach, the scan length was changed from the sella to the sternoclavicular junction, rather than extending to the mediastinum. This approach is contrary to the widespread belief that the mediastinum must be included in all neck CT examinations when evaluating a suspected abscess to exclude mediastinal extension. Of the 27 abscesses seen in this study, none extended to the mediastinum.

An average estimated dose reduction of $47 \%$ supports the hypothesis of this study that decreasing the z-axis length will achieve significant dose savings. This finding is in concordance with the principles of as low as reasonably achievable. Furthermore, children are much more susceptible to the deleterious effects of ionizing radiation than their adult counterparts. In addition, the smaller size of a child leads to a greater amount of energy being imparted to the center of their body than in an adult, consequently increasing organ and effective doses. ${ }^{10}$ This outcome is because the absorbed dose is defined as the energy absorbed per unit mass (measured in grays). The smaller size of a child will impart a greater absorbed dose compared with adults. Therefore, it is important to alter scanning parameters when performing CT scans in children to optimize the radiation dose. Parameters that can be changed to optimize the radiation dose in children are the kilovolt and milliampere. For instance, in our study, the milliampere was increased for heavier patients weighing $>40.5 \mathrm{~kg}$ (350 $\mathrm{mA}$ ), and concordantly, it was decreased in children weighing $<22.5 \mathrm{~kg}$ (200 mA) (Table 3).

Decreasing the scan length is another widely accepted method of reducing the radiation dose, but standardized protocols are often an obstacle to this approach. However, the use of standardized protocols is beneficial in many ways. Creating simpler, easyto-use protocols allows more interoperator independence and provides a consistent presentation of images. ${ }^{11}$ Particularly within the emergency department setting, standardized protocols provide an efficient, often physician-independent method of imaging patients.

The inference of results from our study could lead one to surmise that basic knowledge of the anatomy from the initial topographic image is necessary to appropriately adjust the scan length to the clinical indication. This inference is correct. However, CT technologists perform this task every day on each scan length that is chosen for every routine CT. Furthermore, if alteration of the protocol were to occur, the CT technologist should be able to correctly identify the relevant anatomy on the topographic image to appropriately modify the scan length. According to the American Registry of Radiologic Technologists, the certifying board for CT technologists in the United States, sectional anatomic knowledge is required of CT technologists to successfully pass the required certifying examination. This includes knowledge of the anatomy of the soft-tissue structures in the neck. ${ }^{12}$

The communication between the CT technologist and radiologist should increase before the examination to select the region of interest to be scanned. This would increase the involvement of the technologist and radiologist when performing the examination, thereby decreasing the throughput of the CT scanner, which may be particularly troublesome in busy emergency departments. Furthermore, the need for a radiologist to constantly communicate with the technologist to tailor the protocols after hours could be a burden to many practices and could be impractical in certain circumstances. Therefore, additional investigation would need to analyze the practicality of tailoring the z-axis scan length to the specified indication.

Certain guidelines may alleviate some anxiety by tailoring protocols according to the examination indication:

1) When evaluating an abscess of a nontraumatic etiology, termination of the examination at the sternoclavicular junction would not only reduce the radiation dose, but in teenage girls, it would eliminate direct radiation exposure to breast tissue. Of the 27 neck abscesses, 18 were in teenage girls. If one applied this recommendation to this study, all 18 teenage girls would have 
been saved from having direct radiation exposure to their breast tissue. Furthermore, under Automatic Exposure Control, the milliamperes would increase when scanning thicker body parts due to their increased attenuation of $\mathrm{x}$-rays. Eliminating the thicker regions of a standard neck CT (ie, the upper chest/breast/shoulders) would eliminate the higher milliampere sections. Therefore, the modified DLP would be in the conservative range of the actual DLP because the original CTDI ${ }_{\text {vol }}$ was used in the calculation of the modified DLP. Additionally, in cases of suspected neck abscesses, the cephalad extent of the scan would be to the temporomandibular joint. This was chosen due to the anatomic ease of finding it on the initial prescan topographic image, and at the level of the temporomandibular joint, the entire oropharynx would be included.

2) For evaluation of specific "lumps and bumps," a radiopaque marker at the area of interest can be placed, with the scan performed a few centimeters above and below the marker, as described above. Again, for a palpable lesion $<2 \mathrm{~cm}$, the marker would be placed at the epicenter of the abnormality and the scan would be performed $3 \mathrm{~cm}$ above and below the marker. For lesions $>2 \mathrm{~cm}$, the marker would be placed at the cranial and caudal margins of the lesions, and the scan would be performed $3 \mathrm{~cm}$ above and below the marker. These numbers were chosen due to convenience. They may need to be varied depending on the characteristics of the palpable finding.

A few potential limitations for this new modified approach for obtaining neck CT scans in children are as follows:

1) If a patient moves between the scout image and the scan acquisition, the relevant anatomy may not be fully covered. Although this possibility exists for any CT study, the extra few minutes needed to plan the coverage increases the chance that the child may move, and limited coverage reduces the margin for error.

2) Z-axis-length modification relies on the clinician to specify the purpose of the examination. At times, this can be difficult to elicit, given the complexity of clinical presentations. The retrospective analysis limits assessment of the practicality of modifying $\mathrm{z}$-axis length to the specified indication. A multiinstitutional randomized, prospective trial may be helpful for further analysis in this regard.

\section{CONCLUSIONS}

Decreasing the z-axis length of a neck CT targeted to the clinical indication provides a significant savings in radiation dose while not altering diagnostic ability or management. Using standardized protocols regardless of the indication on neck CTs subjects children to substantially more radiation exposure than necessary.

\section{APPENDIX}

\section{Explanation of Terminology}

Z-axis-directional coordinate oriented along the length in a craniocaudal fashion with respect to the patient.

Effective dose-biologic effect of radiation exposure is expressed in millisieverts, which incorporates exposure of various organs to ionizing radiation and the susceptibility of organs to this exposure. $^{13}$

$\mathrm{CTDI}_{\mathrm{vol}}$-calculated absorbed dose is based on a phantom expressed in milligrays. It is effective for comparing radiation doses for scans of varying techniques (eg, CT chest versus CT head). ${ }^{13}$
Dose-length product is expressed in $\mathrm{mGy} \times \mathrm{cm}$ and is the $\mathrm{CTDI}_{\mathrm{vol}}(\mathrm{mGy}) \times \mathrm{z}$-axis length $(\mathrm{cm}){ }^{13}$

Automatic Exposure Control manages the radiation dose and image quality in a reproducible manner by modification of the tube current (milliampere) to the patient's shape, size, and density.

Minimum milliampere is the minimum setting of the tube current used for milliampere modulation during Automatic Exposure Control.

Maximum milliampere is the maximum setting of the tube current used for milliampere modulation during Automatic Exposure Control. ${ }^{14}$

Kilovolt or kilovolt(peak) is the maximum voltage applied across an $\mathrm{x}$-ray tube. Unlike with milliampere, the radiation dose relationship with kilovolt is nonlinear (eg, increasing the kilovolt from 120 to 140 increases the dose by $40 \%$ ).

\section{REFERENCES}

1. Ludwig B, Foster B, Saito N, et al. Diagnostic imaging in nontraumatic pediatric head and neck emergencies. Radiographics 2010;30: 781-99 CrossRef Medline

2. ACR-ASNR-SPR Practice Parameter for the Performance of Computed Tomography (CT) of the Extracranial Head and Neck. http:// www.acr.org/\%7E/media/ACR/Documents/PGTS/guidelines/CT_ Head_Neck.pdf. Accessed December 15, 2015

3. Huda W. Radiation risks: what is to be done? AJR Am J Roentgenol 2015;204:124-27 CrossRef Medline

4. Pearce MS, Salotti JA, Little MP, et al. Radiation exposure from CT scans in childhood and subsequent risk of leukaemia and brain tumours: a retrospective cohort study. Lancet 2012;380:499-505 CrossRef Medline

5. Meulepas JM, Ronckers CM, Smets AM, et al. Leukemia and brain tumors among children after radiation exposure from CT scans: design and methodological opportunities of the Dutch Pediatric CT Study. Eur J Epidemiol 2014;29:293-301 CrossRef Medline

6. Shah NB, Platt SL. ALARA: is there a cause for alarm? Reducing radiation risks from computed tomography scanning in children. Curr Opin Pediatr 2008;20:243-47 CrossRef Medline

7. Report of AAPM Task Group 23 of the Diagnostic Imaging Council Committee. The Measurement, Reporting, and Management of Radiation Dose in CT. http://www.aapm.org/pubs/reports/rpt_96.pdf. Accessed December 11, 2014

8. Tercier S, Meier R, Vasseur-Maurer S, et al. Huge mediastinal abscess in a 12-month-old child: case report and review of the literature. Internet J Thoracic Cardiovascular Surg 2004;7. http://ispub. com/IJTCVS/7/2/3121. Accessed February 12, 2015

9. Kumar S, Kumar V, Bishnoi A, et al. Non-traumatic anterior mediastinal abscess in childhood. J Indian Assoc Pediatr Surg 2011;16: 75-77 CrossRef Medline

10. Thomas KE, Wang B. Age-specific effective doses for pediatric MSCT examinations at a large children's hospital using DLP conversion coefficients: a simple estimation method. Pediatr Radiol 2008;38:645-56 CrossRef Medline

11. Haggett S, Van Hoorn W. Working with Protocols. http://www. nuclear-medicine.gr/home/viewFile/4. Accessed March 11, 2015

12. ARRT Structured Education Requirements: CT. https://www. arrt.org/pdfs/Structured-Education-Requirements/CT-StructuredEducation-Requirements.pdf. Accessed September 26, 2015

13. Saini S. Multi-detector row CT: principles and practice for abdominal applications. Radiology 2004;233:323-27 CrossRef Medline

14. Brueswitz, MR, Yu L, Vrieze TJ, et al. Smart mA-Automatic Exposure Control (AEC): physics principles and practical hints. In: Proceedings of the Radiological Society of North America 94th Scientific Assembly and Annual Meeting, Chicago, Illinois. November 30 to December 5, 2008 